\title{
Physiological Changes in the Cornea of the Ageing Eye
}

\author{
C. G. WIGHAM and S. A. HODSON \\ Cardiff
}

\begin{abstract}
Summary
Donor human corneas were studied in vitro for the viability of their endothelia. Endothelial ionic permeability (measured by electrical conductivity) and bicarbonate pump activity (measured by short circuit current) were recorded. Paired corneas were used to monitor post-mortem deterioration times and drug therapy immediately prior to death noted, when possible, to identify any possible effect on tissue performance.

Donor age was correlated with some loss of endothelial pump capability. In adults it seems that pump capability deteriorates with a half time decay of 72 years (correlation coefficient, 0.69). Typically, between 60 and 90 years, endothelial pump capability may drop from $32 \mu \mathrm{amps} . \mathrm{cm}^{-2}$ to $22 \mu \mathrm{amps} . \mathrm{cm}^{-2}$, although at any particular age, the natural variation is $\pm 6 \mu \mathrm{mps} . \mathrm{cm}^{-2}$. Over the same period, endothelial permeability does not deteriorate and there is a suggestion that the endothelial cells may even tighten up with ageing, thereby partially compensating for the suggested ageing diminution in metabolic pump capability. There is no obvious age threshold for endothelial deterioration.

The use of paired corneas revealed a 50 per cent loss in endothelial barrier function per 4.8 days of storage, and less than 5 per cent loss of pump capability over a similar period. The eyes were stored whole in a moist chamber at $0-4$ degrees $C$.

A significant group of donor eyes had a markedly reduced pump capability which was not age related. The patients had had terminal drug treatments which included digoxin and furosemide.
\end{abstract}

Corneal physiology is sufficiently well understood, that experimental observations may now be useful in clinical practice. ${ }^{1}$ If we review the properties of the cornea which regulate corneal hydration we find that there are three of major importance: corneal swelling pressure, endothelial permeability and endothelial bicarbonate pump rate. The isolation of these three factors is not a simple matter but requires the mathematics of irreversible thermodynamics, the details of which are beyond the requirements of this paper, however an approximate description can be given.

Corneal stroma has a passive tendency to swell by a net absorption of both water and ions. The net movement of water and ions into or out of the stroma is always coupled under standard conditions. For example, if somehow water were excluded from the stroma there could be no accumulation of ions within it. Similarly if ions were pulled out of the stroma, then water would also flow out of the 




Fig. 1. The pathways of ion and water movement across corneal endothelium (movement into the cell and out on the same face are omitted). Ions traverse endothelium through the paracellular route. The single exception is the addition of a trans-cellular uni-direction 'pump' route for bicarbonate. Electrical conductivity of the endothelium is a measure of the integrity of the paracellular route (the 'leak'). Short circuit current is a measure of the activity of the bicarbonate pump (the 'pump'). Voltage is current divided by conductance and gives an indication of how the 'pump' and 'leak' are balanced.

stroma and the cornea would deturgesce. This obligatory coupling of net ion movement and water does not require the presence of cell membranes.

Although the stroma strives to absorb water and ions, and consequently to swell, the rate of swelling is limited by the bounding cellular membranes, the epithelium and endothelium. As the epithelium presents a much greater barrier than the endothelium, ${ }^{2}$ because the endothelium is much more permeable to water than ions ${ }^{3}$ and because their joint entry must be coupled, the actual rate limiting step to corneal swelling is the ionic permeability of the endothelium.

For transparency, corneal stroma must not swell, any leak of ions into the stroma across the endothelium must be balanced by a metabolically coupled efflux of ions out of the stroma and into the aqueous humour. This is provided by the bicarbonate pump of the endothelium. ${ }^{4}$ The major flows across the endothelium, and their routes, are illustrated in Figure 1.

Recent experiments have shown that the electrical conductivity (C) of corneal endothelium is linearly related to the ionic per- meability $(\mathrm{P})$. of the endothelium in both rabbit and man. ${ }^{5}$ The magnitude of $\mathrm{C}$ is a real indicator of the structural integrity of the endothelial mosaic. It increases only if the paracellular route leaks more or if individual cells drop out of the mosaic. Short circuit current (i) across the endothelium is generated by the active bicarbonate pump. The electrical potential (V) across the endothelium is, from Ohm's law, the ratio of $\mathrm{i} / \mathrm{C}$ and can be taken as a reliable measure of the ratio of the pump (proportional to i) and leak (proportional to $\mathrm{C}$ ) across the endothelium. In rabbits where it is known that in vitro pump-leak balance is very similar to in vivo pump-leak balance, $\mathrm{V}=650 \mu \mathrm{V}$ (lens-side negative). In man we measure a potential of approximately $-450 \mu \mathrm{V}$, in vitro, the measurement cannot be made in vivo. We believe that our in vitro data presented here are likely to be within 10 per cent, or less, of the in vivo values. ${ }^{6}$

With this information available relating electrophysiological measurements to corneal function we made observations on the cornea with reference to changes associated with donor age, storage time of donor cornea and drug therapy at time of death.

\section{Materials and Methods}

Human eyes were removed from cadavers following the normal procedures. Time of death, cause of death, age and any drug therapy prior to death were noted when possible.

Eyes were removed and placed on a stand inside a sealed glass jar kept humid by placing a small pad of cotton wool soaked in 0.9 per cent saline in the corner of the jar. The eyes were either used immediately or refrigerated at $0-4$ degrees $C$ until use.

The corneal epithelium was removed using a rotating bristle brush. The cornea, with approximately $4 \mathrm{~mm}$ of sclera, was mounted between the two half chambers of an Ussing type apparatus. ${ }^{7}$ The chamber design is such that clamping is directly on to the cornea and not the sclera..$^{5}$ Preliminary experiments using human tissue revealed a slight problem in that as the two half chambers clamped together there was a tendency for the tissue to slip, causing a loss of endothelial cells. This was prevented by painting a ring of cyanoacrylate glue on the face of the tear-side half chamber. The preparation was fixed to this and then the second half chamber positioned and clamped.

Having mounted the tissue the two half chambers 




Fig. 2. A time course of spontaneous electrical potential found across an incubated human corneal endothelium. The initial adjustment is a recovery period after which the activity continues for over 8 hours. This was a relatively unstable trace, slowly changing from a peak of $390 \mu \mathrm{V}$ to a trough of $360 \mu \mathrm{V}$ and recovering to a second peak of $405 \mu \mathrm{V}$.

were filled with human Ringer containing: $\mathrm{NaCl}$, $117.5 \mathrm{mM} ; \mathrm{NaHCO}_{3}, 24 \mathrm{mM} ; \mathrm{CaCl}_{2}, 2.5 \mathrm{mM}$; $\mathrm{Na}_{2} \mathrm{HPO}_{4}, 1 \mathrm{mM} ; \mathrm{MgSO}_{4}, 1 \mathrm{mM}$; glucose, 4.45 $\mathrm{mM}$; reduced glutathione, $1 \mathrm{mM}$, and bubbled to $\mathrm{pH} 7.38$ with an 88 per cent $\mathrm{N}_{2}, 7$ per cent $\mathrm{O}_{2}, 5$ per cent $\mathrm{CO}_{2}$ gas mixture. Continuous perfusion and intrachamber stirring maintained the corneas for a minimum of 6 hours with no signs of deterioration.

Methods used to measure the electrical activity across the preparation have been described in detail previously. 5,7 Briefly, electrical potential difference (V) across the tissue is measured continuouisly by means of small sensing tubes positioned close to the tissue, these Ringer filled tubes are connected to polyacrylamide salt bridges in turn connected to a voltmeter (via calomel electrodes). This system gives us a resolution of $\pm 1 \mu \mathrm{V}$.

Electrical resistance $(\mathrm{R})$, or its more commonly used reciprocal conductivity $C=1 / R$, was measured at frequent intervals by passing a known electrical current across the tissue using $\mathrm{Ag} / \mathrm{AgCl}$ electrodes positioned one at each end. of the chamber, and observing the change in $\mathrm{V}$ across the tissue. This value gives only the total $R$, endothelial cell $R(R e)$ is obtained by subtracting the blank resistance from total resistance (blank resistance being the resistance of the preparation with the endothelium removed). Pump activity of the tissue is described by the tissue generated current $i$, calculated using Ohm's law, i = V.C.

\section{Results}

When the electrical potential across isolated human corneal endothelium is measured in vitro at 35 degrees $\mathrm{C}$ it is always found to be of polarity lens side negative. The magnitude increases during the first hour of incubation to a maximum value which is stable over a minimum 6 hour period (Fig. 2). The initial increase is caused by an increase in the activity of the bicarbonate pump as it recovers from storage conditions. The conductance $(=$ permeability) of the endothelium remains essentially stable throughout. Values quoted in this paper are those of the stable maximum period.

We were interested in post-mortem changes in stored corneal endothelia, to allow us to compare corneas from different donors which inevitably have variations in post-mortem duration. To do this, one eye of a pair was monitored immediately it became available, the partner eye was stored for a 3-8 day period before testing. The results are shown in Figure 3. The top graph indicates that endothelial conductivity, C (proportional to ionic permeability) increases with storage and doubles every 4.8 days on average. The middle graph shows that in contrast, $i$, the short circuit current (proportional to the metabolically generated bicarbonate pump), is reasonably constant over the 8 day period of testing.

The ratio of these two values, trans-endothelial electrical potential, $\mathrm{V}(=\mathrm{i} / \mathrm{C})$, consequently diminishes by about one half over 4.8 days. It is possible from these data, to predict 

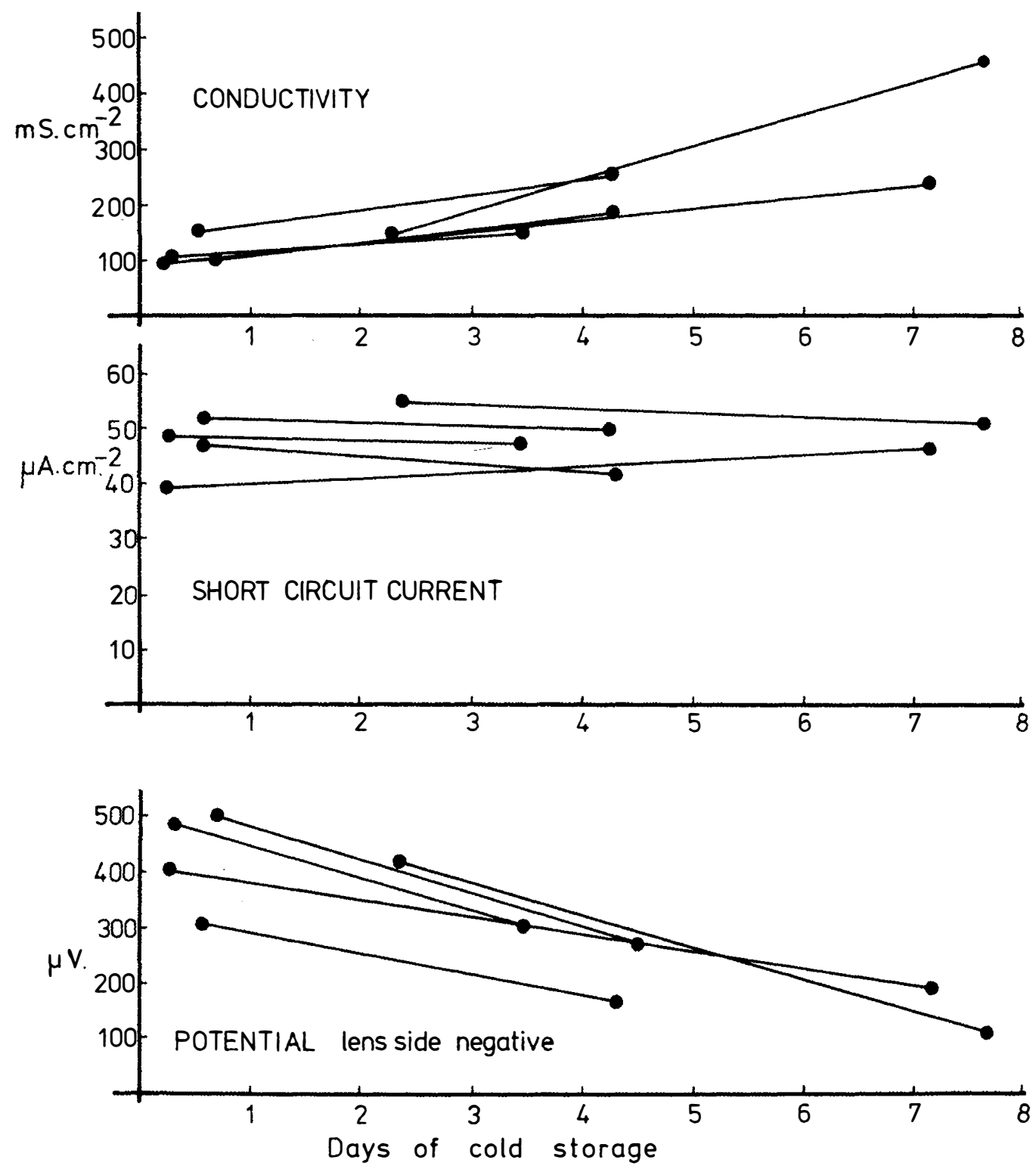

Fig. 3. The effect of post-mortem storage on corneal endothelium. Conductivity (proportional to 'leakiness') increases, whereas short circuit current (proportiona to 'pump') is stable. Consequently electrical potential diminishes. Straight lines each join paired corneas.

how endothelia may vary in different postmortem groups. For example, in corneas collected and tested from $0-12$ hours post-mortem we can expect variations of endothelial electrical potentials of \pm 2.5 per cent; for 12-24 hours, \pm 2.5 per cent and for $24-48$ hours, \pm 5.5 per cent. Comparing corneas collected $0-12$ hours post-mortem with those collected 12-24 hours should show a system reduction of 5 per cent in electrical potential and, if we compare 0-12 hours with 12-24 hours a 16 per cent reduction in potential. 


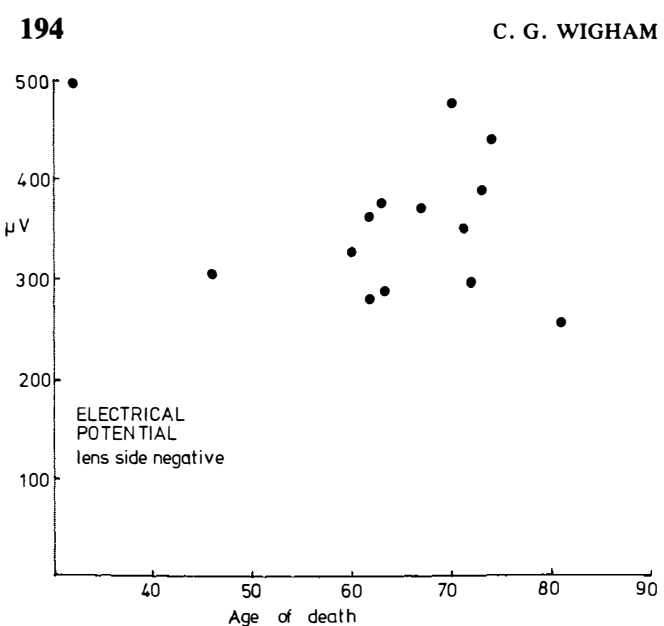

Fig. 4. There is no obvious relationship between age of death and spontaneous electrical potential found across corneal endothelium less than 24 hours postmortem.

Because we felt that systemic errors of 16 per cent were too great we pooled data in this paper only from corneas collected within 24 hours of death.

Figure 4 illustrates post-mortem transendothelial potential difference $(\mathrm{V})$ as a function of age of death in 16 patients. There is no obvious relationship. However, when i (proportional to pump), is plotted against age it can be seen that there is a relationship, with pump diminishing with increasing age (Fig. $5)$. The correlation coefficient $(0.69)$ suggests that in adults the pump may deteriorate with a half life of 72 years. On average the endothelial pump of a $40,50,60,70$ or 80 year old could be expected to generate, in our sample, $38,35,31,28$ or $25 \mu \mathrm{A} . \mathrm{cm}^{-2}$. However, the standard deviation at any particular age would be approximately $\pm 5 \mu \mathrm{A} . \mathrm{cm}^{-2}$. There was a weak correlation (coefficient $=0.29$ ) between donor age and endothelial conductivity (C) (Fig. 6). It is suggested from these data that the endothelial mosaic must tighten up, with respect to ions permeating across it, as it ages. This process seems to be slower than the loss of pump activity seen in Figure 3. Probably the tightening up of the endothelium has a half-life of 200 years but inevitably there is much uncertainty about this value.

One group of donors, which was not included in any of the data reported above, showed consistently lower values of V. It became clear over the years that this investigation took place that donors on a terminal drug regime that included the diuretic furosemide and digoxin had unusually low values of $\mathrm{V}$ (Fig. 7). All these patients died from ischaemic heart disease or myocardial infarction. When compared with an age matched control group, two of whom died after myocardial infarction but were not treated with furosemide or digoxin, it is seen that the difference of endothelial viability is highly significant, $p<0.001$. The group not receiving furosemide/digoxin drug therapy, age $=62.5 \pm 14.9$ years (mean \pm standard deviation) gave a mean value for $\mathrm{V}$ of $366 \pm 23$ $\mu \mathrm{V}$ ( \pm standard error, lens-side negative). The treated group, age $=64.8 \pm 12.6$ years (mean \pm standard deviation) gave a mean $\mathrm{V}$ of $150 \pm 21.9 \mu \mathrm{V}$ (lens-side negative).

\section{Discussion}

To our knowledge, this study, including its preliminary unreported development of methods, is the largest to date on the ageing characteristics of human corneas. Nevertheless, it reports too few cases to be considered definitive, although we believe that the evaluation of each particular cornea is accurate and definitive.

It is a retrospective study of our records, and there can be no experimental design to such investigations, one accepts as a privilege and gratefully whatever corneas are donated, and hopes for sufficient funding to maintain continuously at the ready, a sophisticated and difficult experimental protocol. As it has proved impossible to sustain the juggling act,



Fig. 5. There is a relationship (correlation coefficient $=0.69$ ) between endothelial 'pump' (proportional to current) and age of death. The half life of 'pump' deterioration is about 72 years. 


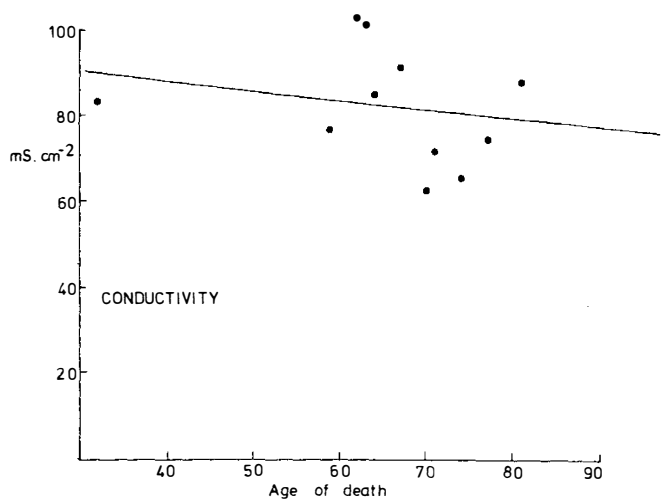

Fig. 6. There is a weak relationship (correlation coefficient $=0.29$ ) between age of death and endothelial 'leakiness' (proportional to conductivity). It seems possible that the endothelium tightens up with age but very slowly. The half life is estimated at around 200 years.

we report our studies here in this preliminary state because of our expectation that we shall be unable to extend our investigations in the future.

The post-mortem changes in individual corneas (Fig. 3) are interesting, they indicate that there is little or no metabolic deterioration in endothelial pump capability with cold storage, although there is considerable deteri-



Fig. 7. Donors terminally treated with the diuretic furosemide (encircled points) developed a much lower trans-endothelial potential on incubation (lower dashed line) than an age matched sample of other donors (higher dashed line). Cause of death: circles-myocardial infarction (note that two of these donors were not treated with furosemide); squares-ischaemic heart disease; crosses-other. oration in endothelial barrier function. We can observe in our stored corneas that the occasional endothelial cell drops out of the mosaic during cold storage, it is likely that this accounts at least in part for the observed drop in barrier function. Sporadic cell loss in the endothelial mosaic seems to be a natural consequence of ageing, the surrounding cells, in vivo, having the capability of making good the deletion. ${ }^{8}$ There is no compelling evidence from these studies to suggest that corneas in whole eye cold storage for a few days should not be made available for keratoplasty. We could not extend our results beyond 8 days cold storage because of collapse of the corneal cone into the anterior chamber at about this time.

Corneal endothelium appears to age by having a diminished pump capability whilst possibly partially compensating by increasing its barrier function and by diminishing its ionic permeability. The permeability changes seem to be very slow and, interestingly, match the reported slow increase in endothelial size, with age. ${ }^{9}$ As it is certain that ions permeate between (and not through) the mosaic of endothelial cells, it is attractive to suggest that the loss of endothelial cells with age may have a physiologically important contribution to maintaining corneal compensation by reducing endothelial permeability. The suggestion from our data is that the game will be lost; the deterioration of pump rate seems faster than the damming effect of permeability change. However, natural ageing compensation should last for a couple of centuries, more than enough for the donor and recipient!

We could identify no age threshold for endothelial deterioration. If the choice is there, however, the younger the donor the better the chance of getting a more vigorous endothelium. Our figures suggest that for example if one were to compare 50 year old donors with 80 year old donors then in one comparison in 6 the 80 year old would have a higher metabolic pump than the 50 year old.

Terminal drug treatment does seem to correlate with a measurable effect on corneal endothelial viability. In particular the diuretic furosemide especially when combined with digoxin appears to be associated with a loss of endothelial pump capability. Although it is 
possible that the cause of the endothelial inhibition lies with the disease itself, the two donors that had myocardial infarctions without furosemide treatment had a significantly higher endothelial function.

It would be interesting to know if there were any clinical implications for the viability of corneas donated from patients on furosemide/digoxin therapy.

We gratefully acknowledge support from the Royal National Institute for the Blind.

\section{References}

${ }^{1}$ Wigham CG, Hodson SA: A test bed for ocular drugs. Ophthal. Physiol. Opt. 1986; 6: 135-7.

${ }^{2}$ Mishima S, Hedbys BO: The permeability of the corneal epithelium and endothelium to water. Exp. Eye Res. 1967; 6: 10-32.
${ }^{3}$ Hodson SA, Wigham CG: Paracellular ionic and transcellular water diffusions across rabbit corneal endothelium. J. Physiol. (In Press).

${ }^{4}$ Hodson SA, Miller F: The bicarbonate ion pump in the endothelium which regulates the hydration of rabbit cornea. J. Physiol. 1976; 263: 563-77.

${ }^{5}$ Hodson SA, Wigham CG: The permeability of rabbit and human corneal endothelium. J. Physiol. 1983; 342: 409-19.

${ }^{6}$ Hodson SA, Wigham CG, Williams L, Mayes M, Graham MV: Observations on the human cornea in vitro. Exp. Eye Res. 1981; 32: 353-60.

${ }^{7}$ Wigham CG, Hodson SA: The, effect of bicarbonate ion concentration on trans-endothelial short circuit current in ox cornea. Curr. Eye Res. 1981; 1: 37-41.

${ }^{8}$ Sherrard ES: The corneal endothelium in vivo: its response to mild trauma. Exp. Eye Res. 1976; 22: $347-57$.

${ }^{9}$ Mishima S: Clinical investigations on the corneal endothelium. Am. J. Ophthalmol. 1982; 93: 1-29. 